\title{
Tuning of Type-1 Servo System Using Swarm Intelligence for SIMO Process
}

\author{
Tushar Jain, Member, IACSIT, IAENG, M.J. Nigam
}

\begin{abstract}
In this paper, the design of servo system is proposed using the state feedback where the parameters of feedback gain matrix are tuned using the particle swarm optimization (PSO). Type-1 servo system is considered as in most of the Single Input-Multi Output (SIMO) process the presence of integrator cannot be identified in the plant. It contains more than one inter-coupled transfer function between the state vectors of any non-linear system. One of the performance objectives for controller design is to keep the error between the controlled output and the set-point as small as possible. The Performance Index (PI) considered are Integral Time Absolute Error (ITAE), Integral Square Error (ISE), Integral Absolute Error (IAE) for optimal control. The simulations are tabulated in section 4 . to analyze the promising results by the proposed method.
\end{abstract}

Index Terms-Particle Swarm Optimization, Performance Index, SIMO, Servo system.

\section{INTRODUCTION}

The type 1 servo system has one integrator in the feed-forward path, and the system will exhibit no steady-state error in the step response. In this paper, the pole-placement approach to the design of type 1 servo system has been carried out. The system is limited to have a scalar control signal $u$ and a scalar output [1]. The block diagram is shown in Figure 1.

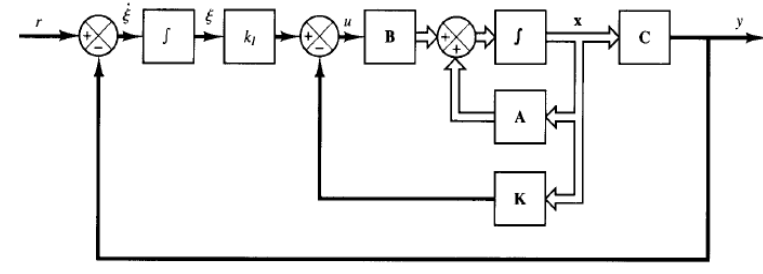

Fig.1 Basic form of Type-1 Servo System

Hence, the system equations are given by:

$$
\begin{aligned}
\overrightarrow{\dot{x}} & =A \vec{x}+B \vec{u} \\
y & =C \vec{x} \\
u & =-\vec{K} \vec{x}+k_{I} \xi \\
\dot{\xi} & =r-y=r-C \vec{x}
\end{aligned}
$$

Manuscript received March 23, 2009.

Tushar Jain, M.J. Nigam, Indian Institute of Technology Roorkee, Uttarakhand, India. Emails: tushaar.iitr@gmail.com(Tushar Jain), mkndnfec@iitr.ernet.in(M.J. Nigam) where $\vec{x}=$ state vector of the plant (n-vector)

$u=$ control signal (scalar)

$y=$ output signal (scalar)

$\xi=$ output of the integrator (state variable)

$\mathrm{r}=$ reference input signal

$\mathrm{A}=n x n$ constant matrix

$\mathrm{B}=n x 1$ constant matrix

$\mathrm{C}=1 x n$ constant matrix

It is assumed that the plant is completely controllable. The basic idea of designing the type- 1 servo system here is to stabilize $(n+1)$ th-order regulator system that will bring the error to zero.

Type-1 servo system can also be designed for the plant having an integrator, though this has not been covered much in detail, as in many non-liner systems the presence of integrator in the SIMO process is difficult to predict before linearization.

Linear Quadratic Regulator (LQR) [2] is designed for optimal control of Inverted Pendulum (IP) using (Genetic Algorithm) GA; the swing-up controller takes approximately 4 seconds to reach the upright position.

\section{PARTICLE SWARM INTELLIGENCE}

Particle Swarm Optimization (PSO) is swarm intelligence based algorithm to find a solution to an optimization problem in a search space, or model and predict social behavior in the presence of objectives [3]. It is a stochastic, population based evolutionary computer algorithm for problem solving. Social influence and social learning enable a person to maintain cognitive consistency.

The swarm is typically modeled by particles in multidimensional space that have a position and a velocity. These particles fly through hyperspace (i.e., $\mathbb{R}^{n}$ ) and have two essential reasoning capabilities: their memory of their own best position and knowledge of the global or their neighborhood's best. For minimizing an optimization problem, "best" simply means the position with the smallest objective value. Members of a swarm communicate good positions to each other and adjust their own position and velocity based on these good positions. So a particle has the following information to make a suitable change in its position and velocity:

1). A global best that is known to all and immediately updated when a new best position is found by any particle in the swarm. 
2). Neighborhood best that the particle obtains by communicating with a subset of the swarm.

The particle position and velocity update equations [4] in the simplest form that govern the PSO are given by:

$\mathrm{v}[]=\mathrm{v}[]+\mathrm{c} 1 * \operatorname{rand}() *($ pbest []$-$ present []$)+\mathrm{c} 2 * \operatorname{rand}()$

* (gbest [] - present [])

present []$=$ present []$+\mathrm{v}[]$

$\mathrm{v}[]$ is the particle velocity, present[] is the current particle (solution). pbest[] and gbest[] are defined as stated before. rand () is a random number between $(0,1)$. c1, c2 are learning factors usually $\mathrm{c} 1=\mathrm{c} 2=2$.

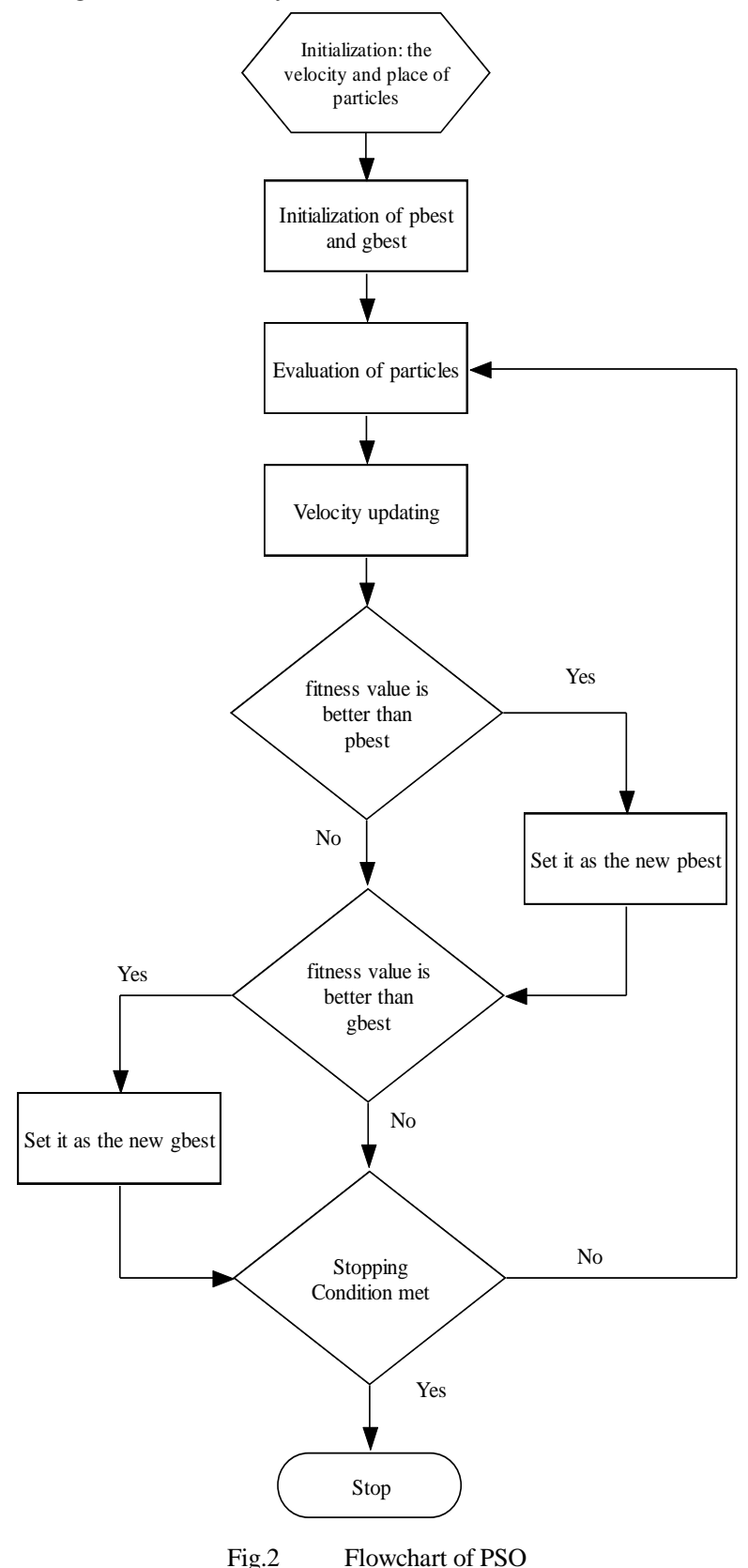

As the swarm iterates, the fitness of the global best solution improves (decreases for minimization problem). It could happen that all particles being influenced by the global best eventually approach the global best, and there on the fitness never improves despite however many runs the PSO is iterated thereafter. The particles also move about in the search space in close proximity to the global best and not
3). The local best, which is the best solution that the particle has seen.

exploring the rest of search space. This phenomenon is called 'convergence'. If the inertial coefficient of the velocity is small, all particles could slow down until they approach zero velocity at the global best. The selection of coefficients in the velocity update equations affects the convergence and the ability of the swarm to find the optimum. One way to come out of the situation is to reinitialize the particles positions at intervals or when convergence is detected. The flowchart of PSO algorithm is shown in Figure 2.

The five basic principles of swarm intelligence are articulated [3] as; First, is the proximity principle: the population should be able to carry out simple space and time computations. Second is the quality principle: the population should be able to respond to quality factors in the environment. Third is the principle of diverse response: the population should not commit its activities along excessively narrow channels. Fourth is the principle of stability: the population should not change its mode of behavior every time the environment changes. Fifth is the principle of adaptability: the population must be able to change behavior mode when it's worth the computational price.

It can be viewed as a mid-level form of A-life or biologically derived algorithm, occupying the space in nature between evolutionary search, which requires eons, and neural processing, which occurs on the order of milliseconds.

\section{SIMO CASE STUDY}

To investigate the impact of PSO on the design of type-1 servo system using state feedback, a nonlinear SIMO process: Inverted Pendulum system is considered.

\section{A. Inverted Pendulum}

The inverted pendulum control problem [5] is usually presented as a pole balancing task. The system to be controlled consists of a cart and a rigid pole hinged to the top of the cart. The cart can move left or right on a one-dimensional bounded track, whereas the pole can swing in the vertical plane determined by the track. The linearized system equations around $\theta=\pi$ in the state space are:

$$
\begin{aligned}
{\left[\begin{array}{c}
\dot{x} \\
\ddot{x} \\
\dot{\theta} \\
\ddot{\theta}
\end{array}\right]=\left[\begin{array}{cccc}
0 & 1 & 0 & 0 \\
0 & \frac{-\left(I+m l^{2}\right) b}{I(M+m)+M m l^{2}} & \frac{m^{2} g l^{2}}{I(M+m)+M m l^{2}} & 0 \\
0 & 0 & 0 & 1 \\
0 & \frac{-m l b}{I(M+m)+M m l^{2}} & \frac{m g l(M+m)}{I(M+m)+M m l^{2}} & 0
\end{array}\right]\left[\begin{array}{c}
x \\
\dot{x} \\
\theta \\
\dot{\theta}
\end{array}\right] } \\
+\left[\begin{array}{c}
0 \\
\frac{I+m l^{2}}{I(M+m)+M m l^{2}} \\
0 \\
\frac{m l}{I(M+m)+M m l^{2}}
\end{array}\right] u
\end{aligned}
$$




$$
y=\left[\begin{array}{llll}
1 & 0 & 0 & 0 \\
0 & 0 & 1 & 0
\end{array}\right]\left[\begin{array}{c}
x \\
\dot{x} \\
\theta \\
\dot{\theta}
\end{array}\right]+\left[\begin{array}{l}
0 \\
0
\end{array}\right] u
$$

Where, $\quad \mathrm{M}=$ mass of cart $=0.5 \mathrm{~kg}$

$$
\begin{aligned}
\mathrm{m} & =\text { mass of pendulum }=0.2 \mathrm{~kg} \\
\mathrm{~b} & =\text { friction of cart }=0.1 \mathrm{~N} / \mathrm{m} / \mathrm{sec} \\
\mathrm{I} & =\text { inertia of pendulum }=0.006 \mathrm{kgm}^{2} \\
\mathrm{l} & =\text { length of pendulum's center }=0.3 \mathrm{~m} \\
\mathrm{~F} & =\text { force applied to cart }
\end{aligned}
$$

The state of the system is defined by values of four system variables: $(x, \dot{x}, \theta, \dot{\theta})$ the cart position, cart velocity, pendulum angle and angular velocity of the pendulum pole, respectively. Control force is applied to the system to prevent the pole from falling while keeping the cart within the specified limits.

Real time hardware of 1-stage Linear Inverted Pendulum manufactured by GoogolTech, China is used for simulation.

\section{RESULTS AND DISCUSSION}

The open loop step response of the pendulum angle and the cart position is shown in Figure 3.

It can be seen that a small force on the cart sets the pendulum angle at $90^{\circ}$ therefore, to improve the dynamics of the system; some controller has to be designed. The Simulink diagram of type-1 servo system using state feedback controller for IP is shown in Figure 4.

The performance of the state feedback controller can be judged by the value of its parameters. The PSO algorithm explained in Section 2. has been used to estimate the parameters of the feedback gain matrix

Performance Index (PI) is optimized for position of the cart as in the real system, the length of the apparatus on which the cart is moving is limited. So, care has been taken to restrict the motion of cart within the limits. This is analyzed on the basis of ISE, IAE and ITAE to maintain the pendulum position at $0^{0}$ for any disturbance given to the cart.

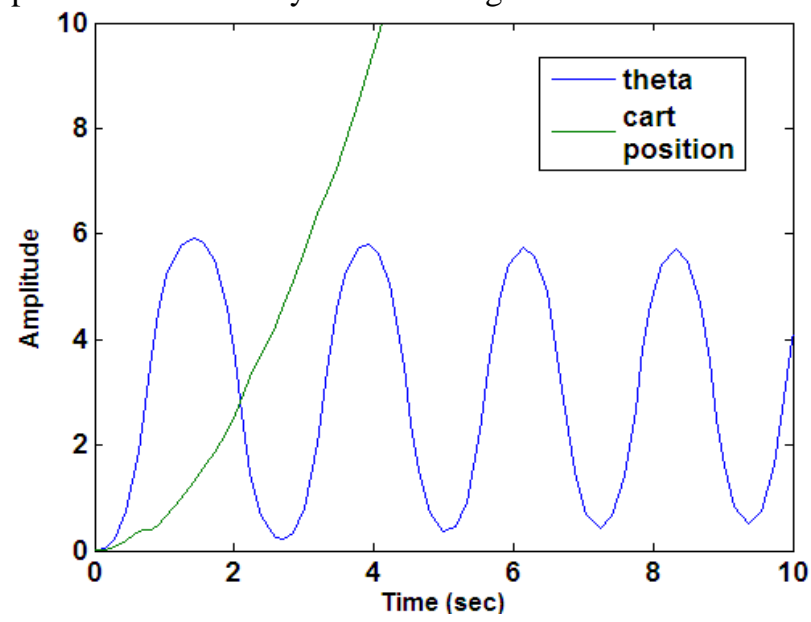

Fig.3 Open loop step response of IP

The simulated results of controller using PSO for different types of errors are shown in Figure 5, Figure 6, and Figure 7.

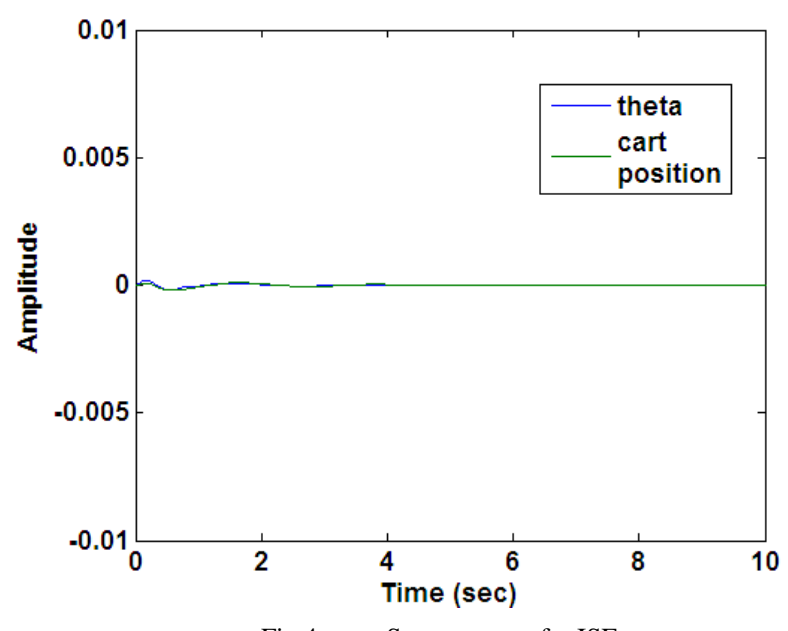

Fig.4 Step response for ISE

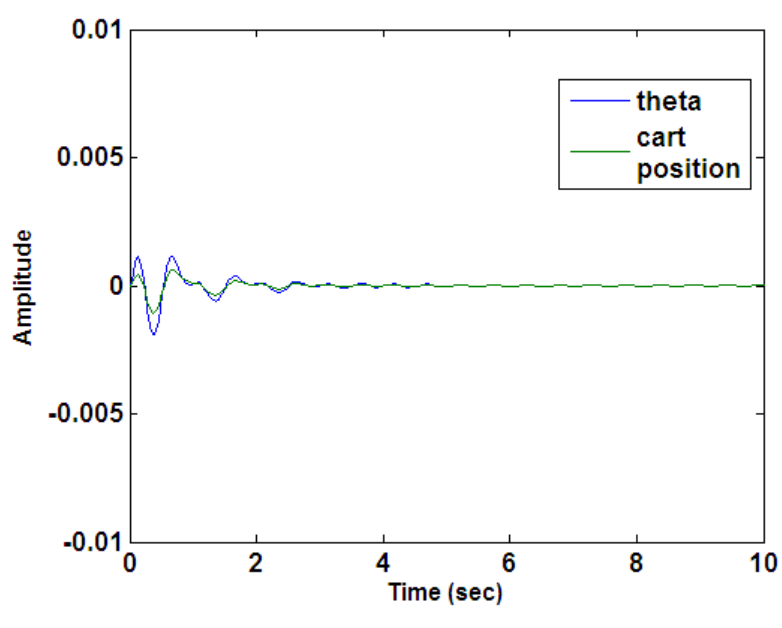

Fig.5 Step Response for IAE

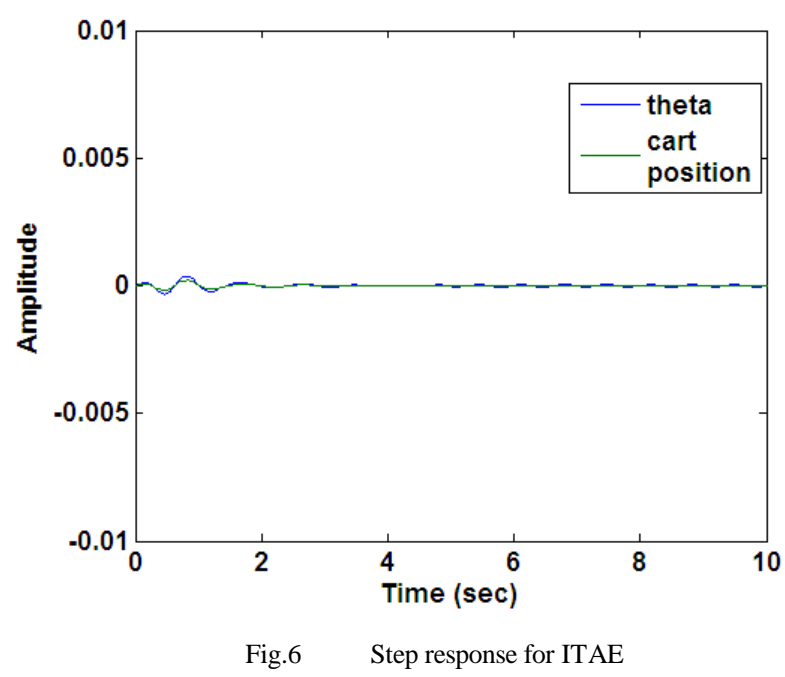

Neuro-PID controller [6, 7] via error-back-propagation (BP) method is designed using GA as optimization technique; it took much time to get convergence results. Also, the 
overshoot of the pendulum angle is large in comparison to techniques discussed above.

TABLE 1. FEEDBACK GAIN MATRIX W.R.T. PI

\begin{tabular}{|l|l|l|l|}
\hline $\begin{array}{l}\text { PERFORMANCE } \\
\text { INDEX }\end{array}$ & $\begin{array}{l}\text { W.R.T. } \\
\text { ISE }\end{array}$ & $\begin{array}{l}\text { W.R.T. } \\
\text { IAE }\end{array}$ & $\begin{array}{l}\text { W.R.T. } \\
\text { ITAE }\end{array}$ \\
\hline FITNESS VALUE & 0.000083 & 0.01363 & 0.008278 \\
\hline K1 & -35.217 & -47.7867 & -117.6538 \\
\hline K2 & -51.453 & -28.3160 & -159.5329 \\
\hline K3 & 17.096 & 111.3929 & 51.1659 \\
\hline K4 & 71.769 & 57.0990 & 124.0727 \\
\hline KI & -191.633 & -702.5304 & -1513.3 \\
\hline
\end{tabular}

\section{CONCLUSION}

For the SIMO process under consideration, type-1 servo system proves to be a promising controller employing PSO algorithm as compared to [2], [6]-[7]. Figure 5, 6, and 7 shows the effectiveness of the proposed method for single stage Linear Inverted Pendulum system. As it is analyzed, a single PID controller cannot be used with real system and employing two PID controllers for controlling cart position and pendulum angle, requires much computation time for number of iterations.

While designing state feedback controller, the computation time is less using search algorithm for the optimal feedback gain matrix. In PSO, the search region is independent of the boundary condition specified by the number of parameters to be tuned, it is given by the distance between the randomly selected initial position and position corresponding to optimal fitness value.

PSO algorithm combined with other intelligent techniques, such as neural networks, expert systems, and fuzzy logic control systems open a new way to design and construct control systems adapted to complex process.

\section{REFERENCES}

[1] Katsuhiko Ogata, Modern Control Engineering, Prentice Hall, New Jersey, $3^{\text {rd }}$ edition- 1997

[2] Hamid R. P., M. R. Jaheh-Motlagh, Ali-Akbar J., "Optimal feedback control design using genetic algorithm applied to inverted pendulum", IEEE International Symposium on Industrial Electronics, pp. 263-268, June, 2007

[3] James Kennedy, Russell Eberhart, "Particle Swarm Optimization", IEEE Transactions, pp. 1942-1948, 1995

[4] Qi Kang, Lie Wang, Qi-di Wu, "Research on Fuzzy Adaptive Optimization Strategy of Particle Swarm Algorithm", International Journal of Information Technology. pp. 65-77, Vol.12, No.3, 2006

[5] Dadios, Elmer P.; Williams, David J., "A Fuzzy-Genetic Controller for the Flexible Pole-Cart Balancing Problem", Proceedings of IEEE International Conference on Evolutionary Computation, pp 223, ISBN: 078032902 3, Nagoya, Japan, 1996.

[6] Sigeru Omatu, Safaai Deris, "Stabilization of Inverted Pendulum by the Genetic Algorithm", Proceedings of IEEE, pp. 700-705, 1996.

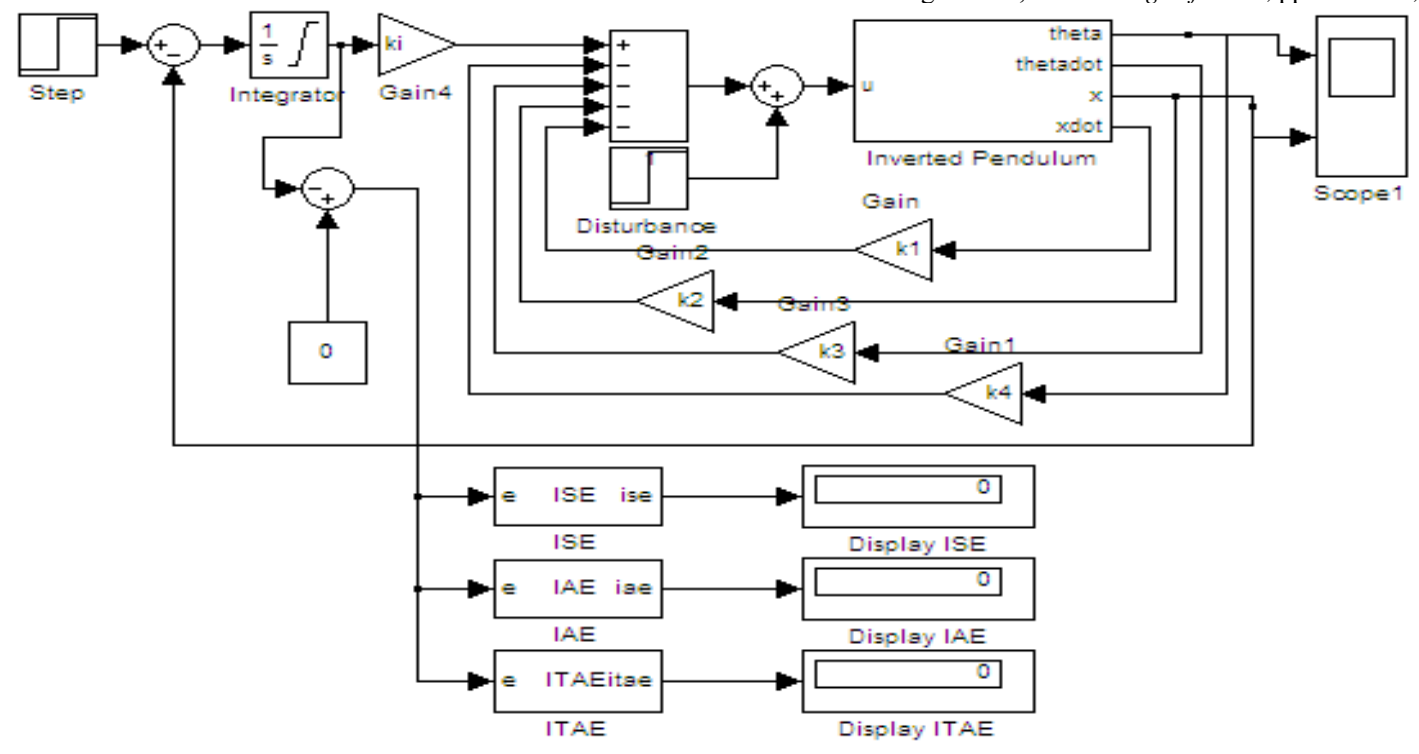

Fig.4 Simulink block of Inverted Pendulum 